VOL. I (1969), 209-212

\title{
Interior preserving maps
}

\section{T. Memp}

It is a curious fact that, while the functions which preserve closures are exactly the closed continuous functions, the interior-preserving functions form a much smaller class than the open continuous functions. In fact, the interior-preserving maps are essentially homeomorphisms, surjections between discrete spaces, and "sums" of these.

It is well-known that a function $f: X+Y$ is closed and continuous if and only if it preserves closures (i.e. $\operatorname{Cl} f[A]=f[\mathrm{ClA}]$ for all $A \subseteq X$ ). The analogous statement for interiors is not true. Indeed (as we shall show) the class of interior preserving maps is far more restrictive than the class of open continuous maps. Clearly any homeomorphism as well as any function from a discrete space to a discrete space is interior preserving. A curious fact (cf. Corollary to Theorem 2) is that in the Hausdorff case, these types of maps and their combinations are the only interior preserving maps.

DEFINITION. A function $f: X \rightarrow Y$ is said to preserve interiors provided that Int $f[A]=f[$ Int $A]$ for all $A \subseteq X$.

To see that "interior preservation" does not characterize the open continuous functions, notice that the projection of the closed unit square onto the closed unit interval is not only open and continuous but is closed and proper as well, yet it does not preserve interiors. The following theorem yields the necessary characterization.

Received 21 March 1969. Received by J. Austral. Math. Soc. 30 September 1968. Revised 18 November 1968. Communicated by G.B. Preston. The author wishes to express his appreciation to the referee for the substantial improvements suggested by him. 
Let $f: X \rightarrow Y$ be any surjection. We call any (not necessarily continuous) function $\varepsilon: y \rightarrow K$ such that $f \cdot s=$ identity, a section of $f$. Clearly, any choice $s(y) \in f^{-1}(y)$ for each $y \in Y$ provides a section of $f$.

THEOREM 1. Let $f: X \rightarrow Y$ be a function on topological spaces. The following three properties are equivalent:

1. $f$ preserves interiors.

2. Every section $s: f[X] \rightarrow X$ of $f$ is an embedding of $f[X]$ onto an open subset of $X$.

3. $f$ is continuous; and $f[V]$ is open in $Y$ if and only if $V$ is open in $X$.

Proof. $1 \Rightarrow 2$. Let $s$ be any section of $f$. We first show that $s$ is an open map. Let $W \subseteq f[X]$ be open; then

$$
f[\text { Int } s[W]]=\operatorname{Int} f[s[W]]=\operatorname{Int} W=W=f[s[W]] \text {; }
$$

since Int $s[W] \subseteq s[W]$ and $f \mid s[W]$ is injective, this shows that $s[W]=$ Int $s[W]$, so $s[W]$ is open in $X$. Thus, $s$ is an open map and, in particular, $s[f[X]]$ is open in $X$.

Let $V$ be open in $X$. Then

$$
s^{-1}[V]=f \cdot s\left[s^{-1}[V]\right]=f\left[s \cdot s^{-1}[V]\right]=f[V \cap s[f[X]]] .
$$

Here, $V \cap s[f[X]]$ is open in $X$, so $f[V \cap s[f[X]]]=$ Int $f[V \cap s[f[X]]]$; thus $s^{-1}[V]$ is open and $s$ is continuous. Since $s$ is infective, $s: f[X] \rightarrow s[f[X]]$ is a homeomorphism.

$2 \Rightarrow 3$. Let $V$ be open in $X$. Choose a section $s: f[X] \rightarrow X$ with the property that $s(y) \in V$ for each $y \in f[V]$. Then $f[V]=s^{-1}[V]$ and therefore $f[V]$ is open in $Y$.

Let $f[V]$ be open in $Y$. Given $x \in V$, choose a section $s: f[X] \rightarrow X$ such that $s(y) \in V$ for each $y \in f[V]$ and $s(f(x))=x$. Then $x \in s[f[V]] \subseteq V$ and, since $s[f[V]]$ is open in $X$, this shows that $V$ is open in $X$.

Finally, let $W \subseteq Y$ be open; since $f\left[f^{-1}[W]\right]=W$ is open, we find 
that $f^{-1}[W]$ is open, and $f$ is therefore continuous.

$3 \Rightarrow 1$. Since $f$ is open, we have $f[\operatorname{Int} A] \subseteq \operatorname{Int} f[A][1$, p. 87]. To establish the converse inclusion, for each $y \in$ Int $f[A] \subseteq f[A]$ choose an $a_{y} \in A$ such that $f\left(a_{y}\right)=y$. Let

$$
B=\left\{a_{y} \mid y \in \operatorname{Int} f[A]\right\} \text {. }
$$

Then $B \subseteq A$; and $B$ is open because $f[B]=$ Int $f[A]$. Thus, $B \subseteq$ Int $A$ and

$$
\text { Int } f[A]=f[B] \subseteq f[\operatorname{Int} A] \text {. }
$$

COROLLARY. An injection $f: X \rightarrow Y$ preserves interiors if and only if $f$ is an embedding and $f[X]$ is open.

EXAMPLE. Let $X$ be a sequence which converges to two points. Let $f$ be the quotient map which identifies these two points and is the identity elsewhere. Then $f$ preserves interiors.

Below we show that no interior preserving map can differ radically from the one above; i.e., each such function has its domain partitioned into a discrete part and an open part on which the function is an embedding.

THEOREM 2. Let $f: X \rightarrow Y$ be an interior-preserving surjection, and let

$$
E=\left\{y \mid \operatorname{card} f^{-1}(y) \geqq 2\right\}
$$

Then:

(1) $X-f^{-1}[E]$ and $Y-E$ are open, and $f$ is a homeomorphism of $X-f^{-1}[E]$ and $Y-E$.

(2) $f^{-1}[E]$ and $E$ are discrete.

Moreover,

(3) If $X$ is Hausdorff, then $Y$ is Hausdorff, and $E$ and $f^{-1}[E]$ are open discrete subsets.

Proof (1). Choose sections $s_{1}, s_{2}: Y \rightarrow X$ such that $s_{1}(y) \neq s_{2}(y)$ for each $y \in E$. Then $s_{1}[Y] \cap s_{2}[Y]$ is open in $X$, and is precisely $x-f^{-1}[E]$. Since $f \mid\left[S-f^{-1}[E]\right]$ is continuous, open, and bijective 
to $Y-E$, it is a homeomorphism.

(2). It suffices to show that each $x \in f^{-1}[E]$ is open in $f^{-1}[E]$. Note first that $\{x\} \cup s_{1}[Y]$ and $\{x\} \cup s_{2}[Y]$ have open images in $Y$; consequently these are open sets in $X$. Their intersection

$\{x\} \cup\left[X-f^{-1}[E]\right]$ is therefore open in $X ;$ consequently $\{x\}$ is open in $f^{-1}[E]$. Furthermore $\{f(x)\} \cup[Y-E]$ is open in $Y$, so $\{f(x)\}$ is open in $E$, and therefore $E$ is also discrete.

(3). Since each section is an embedding, $y$ is Hausdorff. Since $E=\left\{y \mid s_{1}(y) \neq s_{2}(y ;\}\right.$ and $s_{1}, s_{2}$ are continuous, it follows that $E$ is open in $Y$ and therefore also $f^{-1}[E]$ is open in $X$.

COROLLARY. Let $X$ be Hausdorff. A surjection $f: X \rightarrow Y$ is interior-preserving if and only if there exist partitions $X=X_{1} \cup D_{1}, Y=Y_{1} \cup D_{2}$, where $X_{1}, Y_{1}$ are open and $D_{1}, D_{2}$ are open discrete, such that $f \mid X_{1}$ is a homeomorphism of $X_{1}$ and $Y_{1}$, and $f \mid D_{1}: D_{1} \rightarrow D_{2}$ is surjective.

Proof. If $f: X \rightarrow Y$ is interior preserving, this holds by Theorem 2. If the conditions hold, then every section of $f$ is an embedding onto an open subset; consequently $f$ is interior-preserving.

COROLLARY. Every interior preserving surjection from a Hausdorff space to a connected space is either a homeomorphism or a constant map with discrete domain.

\section{Reference}

[1] James Dugundji, Topology (Allyn and Bacon, Boston, 1966).

Gainesville,

Florida, USA 